\title{
Organische und funktionelle Durchblutungsstörungen der Hand - differenzialdiagnostische Aspekte
}

\section{Organic and functional circulatory disorders of the hand - aspects of differential diagnosis}

Autor

Uwe Wahl

Institut

BG Klinikum Bergmannstrost Halle, Medizinische Klinik, Halle

\section{Schlüsselwörter}

Hypothenar-Hammer-Syndrom, Vibrationssyndrom,

Berufskrankheit, Raynaud-Phänomen, akrale

Durchblutungsstörungen

\section{Key words}

hypothenar hammer syndrome, occupational disease, hand-arm vibration syndrome, Raynaud's phenomenon, acral circulatory disorders

\section{Bibliografie}

DOI https://doi.org/10.1055/a-1170-9250

Online-Publikation: 28.5.2020

Phlebologie 2020; 49: 233-241

(c) Georg Thieme Verlag KG, Stuttgart · New York

ISSN 0939-978X

Korrespondenzadresse

Dr. med. Uwe Wahl

BG Klinikum Bergmannstrost Halle

Medizinische Klinik

Dir.: Dr. med. F. Ernst, Merseburger Str. 165, 06112 Halle

uwe.wahl@bergmannstrost.de

\section{ZUSAMMENFASSUNG}

Die Abklärung von venösen und arteriellen Durchblutungsstörungen sowie Lymphödemen gehört zur täglichen Praxisroutine des Gefäßmediziners. Nicht selten werden von chirurgischen Kollegen Patienten mit Beschwerden im Schulter-, Arm- und Handbereich zum Ausschluss einer vaskulären Ursache vorgestellt. Der wohl häufigste Abklärungsgrund im Handbereich ist das Raynaud-Phänomen. Neben arteriosklerotischen Durchblutungsstörungen und der Thrombangiitis obliterans sind das Hypothenar-/Thenar-Hammer-Syndrom und das Vibrationssyndrom in den Fingern eher wenig bekannte Entitäten, deren Kenntnis jedoch neben dem medizinischen Vorgehen auch versicherungsrelevante Konsequenzen haben kann. Während beim Hypothenar-/Thenar-Hammer-
Syndrom eine organische Durchblutungsstörung vorliegt, beinhaltet das Vibrationssyndrom der Finger eine funktionell vaskuläre sowie eine nervale Komponente, die eine Trennung vom Karpaltunnelsyndrom erschwert. Bei beiden Entitäten ist eine Berufsanamnese häufig hilfreich. Schon der Verdacht auf eine Berufskrankheit muss nach Gesetzeslage zur Anzeige gebracht werden. Der Artikel beschreibt die wesentlichen pathophysiologischen Mechanismen beider Entitäten und stellt diagnostische und therapeutische Optionen dar. Die Kenntnis beider Erkrankungen erweitert die differenzialdiagnostische Vorgehensweise in der täglichen Praxisroutine bei Patienten mit Schulter-Arm-Hand-Beschwerden und bei Patienten mit Raynaud-Phänomen.

\section{ABSTRACT}

Diagnosing venous or arterial circulatory disorders and lymphedema is a part of the daily clinical routine of vascular physicians. It is not uncommon for surgeons to refer patients with complaints in the arm, shoulder and hand to rule out a vascular cause. Raynaud's phenomenon is probably the most frequent disorder requiring clarification. In addition to arteriosclerosis and thrombangiitis obliterans, knowledge of the somewhat lesser known disorders of hypothenar/thenar hammer syndrome and hand-arm vibration syndrome in the fingers can be important for the medical procedure as well as have insurance-related consequences. While hypothenar/ thenar hammer syndrome is an organic circulatory disorder, hand-arm vibration syndrome in the fingers has a functional vascular component as well as a nerve component, which makes it difficult to differentiate from carpal tunnel syndrome. Knowledge of the patient's occupational history is frequently helpful in diagnosing both disorders. As soon as an occupational disease is suspected, it must be reported by law. This paper describes the primary pathophysiological mechanisms of both disease entities and presents diagnostic and therapeutic options. Knowledge of the two diseases broadens the differential diagnostic approach used in daily practice routines for patients with complaints of the shoulder, arm and hand, or Raynaud's phenomenon. 


\section{Einleitung}

Die Abklärung von Beschwerden in der oberen Extremität in Form von Schwellungszuständen, Schmerzen und Kribbelparästhesien gehört zu der alltäglichen Routine in einer gefäßmedizinischen Praxis. Während im venösen Gefäßsystem Thrombosen (Pagetvon-Schroetter-Syndrom) und im Lymphgefäßsystem chirurgische oder onkologische Eingriffe (v. a. Mammakarzinom) häufigste Ursachen für die klinische Beeinträchtigung sind, kann man im arteriellen Gefäßsystem funktionelle und organisch fixierte Durchblutungsstörungen unterscheiden. Im Besonderen sind Abklärungen von Handbeschwerden wie ein Raynaud-Phänomen oder sensoneurologische Auffälligkeiten im akralen Bereich zu nennen. Nicht selten werden diese Patienten von chirurgischen Kollegen vorgestellt, um vaskuläre Ursachen im Rahmen der Diagnostik eines Karpaltunnelsyndroms oder muskuloskelettale Störungen auszuschließen. Aus der Sicht des Gefäßmediziners sind neben arteriosklerotischen Durchblutungsstörungen oder der Thrombangiitis obliterans das Hypothenar- bzw. Thenar-Hammer-Syndrom (HHS) und die vibrationsbedingten Durchblutungsstörungen der Hand von Bedeutung. Ein einwandfreier Kraft- und Präzisionsgriff ist vor allem in handwerklichen und künstlerischen Berufen unabdingbar. Da bestimmte Krankheitsentitäten mit erheblichen Einschränkungen auf dem Arbeitsmarkt für die Betroffenen einhergehen können, sind sie in den meisten Ländern als Berufserkrankung anerkannt [1]. Neben den therapeutischen lassen sich auch versicherungsrechtliche Konsequenzen aus einer korrekten Diagnosestellung ableiten.

\section{Pathophysiologische und epidemiologische Aspekte bestimmter Krankheitsbilder}

\section{Das Hypothenar-(HHS)/Thenar-Hammer-Syndrom (THS)}

Die Arteria ulnaris speist im Handbereich den oberflächigen Hohlhandbogen und versorgt über die Aa. digitales palmares communes den 3.-5. Finger. Die Arteria radialis ist für die Versorgung des Daumens und Zeigefingers über die Arteria princeps pollicis verantwortlich. Der radial gebildete tiefe Hohlhandbogen dient vorwiegend der metakarpalen Versorgung (Aa. metacarpales palmares). Beide Hohlhandbögen kommunizieren in ca. einem Drittel der Fälle über den Ramus palmaris superficialis der $A$. radialis und den Ramus palmaris profundus der A. ulnaris. Es existieren Bereiche im Verlauf der A. radialis et ulnaris, die aufgrund ihrer anatomischen Lage für einer von außen ausgeübten Gewalteinwirkung und einen Gefäßschaden besonders prädisponiert sind ( $>$ Tab. 1).

Stumpfe Verletzungen führen zu einem Schaden der Tunica intima, sodass subendotheliale Gefäßstrukturen eine Gerinnungsaktivierung und einen Gefäßverschluss bewirken. Einmalig stumpfe Verletzungen können einen thrombotischen Gefäßverschluss verursachen. Wiederholte Gewalteinwirkungen führen zu einer aneurysmatischen Aufweitung des Gefäßes ( $\triangleright$ Abb. 1), die eine Gefahr für thromboembolische Ereignisse bürgt.

Durch Untersuchungen an Patienten mit Raynaud-Phänomen, an dem 5-20\% der Normalbevölkerung leiden, konnten Prävalen-
- Tab. 1 Prädisponierende Lokalitäten für einen Gefäßschaden.

\begin{tabular}{|l|l|}
$\begin{array}{l}\text { A. radialis (Thenar- } \\
\text { Hammer-Syndrom) }\end{array}$ & $\begin{array}{l}\text { A. ulnaris (Hypothenar- } \\
\text { Hammer-Syndrom) }\end{array}$ \\
\hline $\begin{array}{l}\text { Bereich Os scaphoideum/ } \\
\text { Os trapezium }\end{array}$ & Guyon-Loge \\
\hline Ossa metacarpales II/III & \\
\hline
\end{tabular}

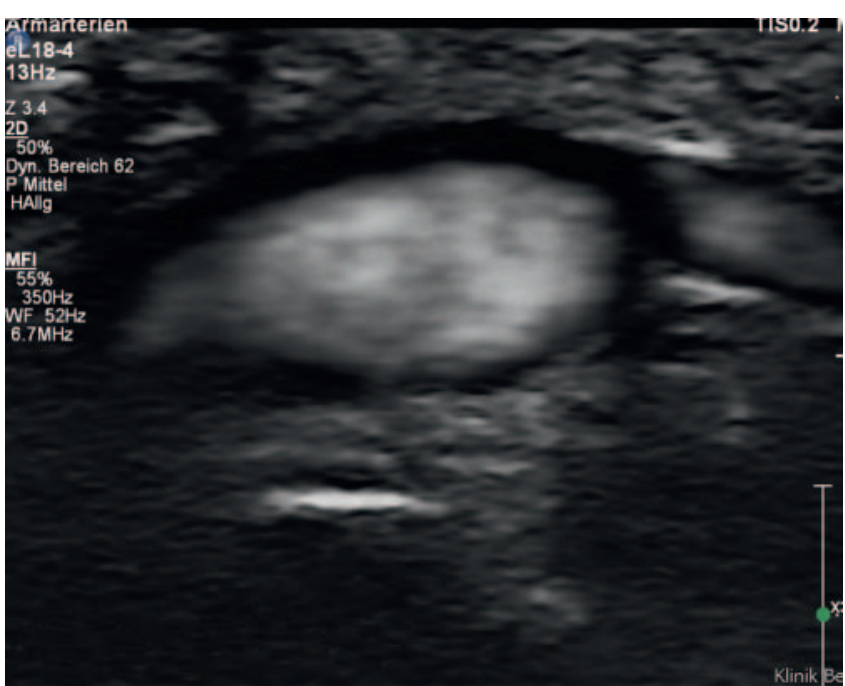

- Abb. 1 Darstellung einer aneurysmatischen Erweiterung der A. ulnaris in der Guyon-Loge (12 mm Länge und $7 \mathrm{~mm}$ im Durchmesser (Vgl. Gegenseite $3 \mathrm{~mm}$ )) im Colour Power Angio Mode (Philips Epiq7).

zen des HHS von 1,13-1,70\% [2-5] ermittelt werden. Die Daten sprechen dafür, dass der Anteil der HHS/THS-Patienten in der Gesamtpopulation $<1 \%$ beträgt.

\section{Vibrationsbedingte Durchblutungsstörungen}

Im Frequenzbereich von 8-1000 Hz treten schädigende Veränderungen an Knochen, Gelenken sowie Gefäßen und Nerven im Schulter-Arm-Hand-Bereich ein [6]. Der Frequenzbereich $\leq 25 \mathrm{~Hz}$ wird hauptsächlich von Armen, Schulter, Nacken und Kopf übertragen und adsorbiert mit der Folge einer Arthrose im Akromioklavikulargelenk und der Osteochondrosis dissecans im Ellenbogengelenk. Höhere Frequenzen mit einem Hauptbereich von 25-500 Hz wirken sich auf Handgelenk und Hand aus (vasospastisches Syndrom, Karpaltunnelsyndrom, Lunatummalazie, Kahnbeinpseudoarthrose) [7, 8]. Das vasospastische Syndrom (besser Vibrationssyndrom der Finger) beinhaltet eine Durchblutungsstörung und eine Nervenschädigung.

Die pathophysiologischen Mechanismen der vibrationsinduzierten Durchblutungsstörung sind bisher nicht ausreichend geklärt [9]. Es handelt sich um eine funktionelle Durchblutungsstörung als Folge einer reflexartigen Vasokonstriktion. Nach einer chronischen Vibrationsexposition kommt es zu Verletzungen der Gefäßinnenhaut, die nicht selten durch zusätzliche Scherkräfte begünstigt wer- 
den [10]. In Untersuchungen konnte gezeigt werden, dass Arbeiter mit chronischer Vibrationsexposition folgende laborchemische Auffälligkeiten aufwiesen, die die Vasokonstriktion unterhalten: erhöhte Spiegel an Endothelin sowie von-Willebrandt-Faktor und Thrombomodulin. Folgend wird die Thrombozytenmigration erhöht, was eine zusätzliche Freisetzung von Thromboxan A2 als gefäßverengendes Hormon verursacht [11-13]. Eine späte Folge ist die Hypertrophie der glatten Gefäßmuskulatur und konsekutive Erhöhung des peripheren Strömungswiderstands. Es kommt somit zu einer Funktionsstörung des Endothels (endotheliale Dysfunktion), die auch an der nicht betroffenen oberen Extremität nachzuweisen ist. Im Vollbild der Erkrankung bestehen systemische Veränderungen (Zunahme alpha-2-adrenerge Rezeptoren, Transmitterpersistenz, Abnahme von alpha-1-adrenergen Rezeptoren) mit komplexen Dysfunktionen im zentralen und autonomen Nervensystem [11]. Die nervalen Schädigungen sind durch demyelinisierende Prozesse gekennzeichnet und betreffen die Thermozeption, Nozizeption sowie die Mechanozeption [14, 15].

Der Anteil der europäischen Arbeiter mit Vibrationsexposition schwankt zwischen 14 und $34 \%$ und erreicht im Bausektor bis zu $63 \%$ [16]. Untersuchungen in tropischen und subtropischen Ländern führen zu dem Schluss, dass die Perfusionsstörungen im Rahmen einer Hand-Arm-Vibrationsbelastung maßgeblich auch von den thermischen Umweltbedingungen abhängen. Su et al. konnten belegen, dass unter wärmeren klimatischen Bedingungen v. a. neurologische Pathologien die Symptomatik prägen [17]. Eine italienische Verlaufsuntersuchung von 249 Vibrationsexponierten ergab eine Prävalenz an einem Vibrationssyndrom mit Fingerabblassung nach 3 Jahren von 10,8\%; erstmalig war die Symptomatik nach durchschnittlich 2-jähriger Exposition bemerkt worden [18]. Andere Studien erbrachten durchschnittliche Expositionszeiten von ca. $11(n=271)$ bis 20 Jahren $(n=165)$, bis sensoneurologische oder vaskuläre Symptome auftraten [19, 20].

\section{Andere akrale Durchblutungsstörungen}

Die Thrombangiitis obliterans (TA) ( $\mathbf{A b b}$. 2) ist eine schubweise verlaufende entzündliche Gefäßerkrankung [21]. Hauptrisikofaktoren sind der chronische Nikotinabusus und das männliche Geschlecht vor dem 50. Lebensjahr. Über immunpathologische Gefäßwandprozesse resultieren thrombotische Verschlüsse, sodass entweder Kollateralen oder auch Teil-/Komplettreperfusionen ehemals verschlossener Arterien (Matorell-Zeichen) ersichtlich sind [22, 23]. Charakteristisch sind akrale Läsionen nach Bagatellverletzungen, die einen Mischbefund aus Perfusionsstörung und Inflammation darstellen [24]. Die PAVK zeigt eher selten einen isolierten Befund im Bereich der Hände und tritt häufig mit arteriosklerotischen Veränderungen der A. subclavia auf. Embolische Komplikationen bei Vorhofflimmern kommen ebenso vor. Vaskulitiden sind Raritäten.

Der häufigste Vorstellungsgrund in der gefäßmedizinischen Praxis im Bereich der oberen Extremität wird die Abklärung eines Raynaud-Phänomens, einer akralen Schmerzsymptomatik oder einer akralen Läsion sein. Im Folgenden soll auf das HHS und THS sowie das Vibrationssyndrom der Finger fokussiert werden, da für diese Entitäten die Kenntnis oft nur marginal ist.

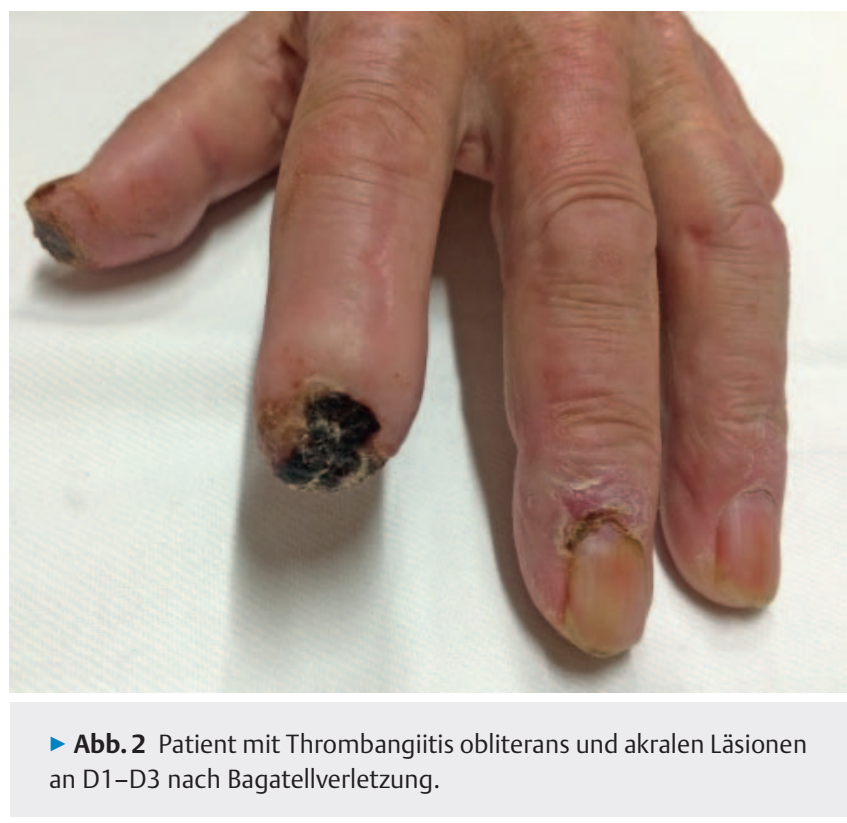

\section{Diagnostische Herangehensweise}

\section{Kritische organische Durchblutungsstörung}

Zunächst muss eine kritische Extremitätenischämie ausgeschlossen werden. Neben der üblichen klinischen Untersuchung mit Inspektion (Haut-, Fingernagel- und Muskelstatus) und Pulsstatus sollte ein Allen-Test erfolgen. Da dieser sehr untersucherabhängige Testergebnisse erbringen kann, wird die Objektivierung unter Hinzunahme der Doppler- oder Duplexsonografie empfohlen [25]. Die akrale optische Pulsoszillografie kann bereits eine organische Durchblutungsstörung (HHS, THS, TA, arterielle Embolie, PAVK) detektieren. Ein unauffälliger Oszillographiebefund schließt jedoch eine relevante Durchblutungsstörung nicht aus weil Gefäßverschlüsse komplett über das Hohlhandbogensytem kompensiert werden können und aneurysmatische Gefäßerweiterungen die Pulsatilität des freiwegigen Gefäßssystems nicht beeinflussen müssen ( $\triangleright$ Abb. 3).

Es muss bei jedem Patienten aus diesem Grund eine duplexsonografische Untersuchung erfolgen. Hochfrequente Linearschallköpfe sind hierfür gut geeignet und heutzutage ubiquitär vorhanden. Eine Arbeitsgruppe konnte Durchmesser für die A. ulnaris bei gesunden Frauen von 1,8 $\pm 0,32 \mathrm{~mm}$ und bei gesunden Männern von 2,2 $\pm 0,46 \mathrm{~mm}$ nachweisen [5].

Kann eine organische Durchblutungsstörung mit den genannten diagnostischen Mitteln nicht ausgeschlossen werden oder macht eine weitergehende Intervention dies erforderlich, sind Schnittbilduntersuchungen oder Angiografien durchzuführen. Die Limitierung von CTA und MRA liegt in der Feinauflösung im akralen Bereich. Die Hypothenarregion kann gut beurteilt werden und mögliche Zusatzinformationen (Knochenbeurteilung, Weichteilapparat) zur Sonografie erbringen. Die digitale Subtraktionsangiografie hat hingegen im akralen Bereich einen hohen diagnostischen Stellenwert. Zudem lassen sich im gleichen Schritt therapeutische Maßnahmen planen oder durchführen. Aus der eigenen Erfahrung ergibt sich nicht selten das Problem eines akralen Vasospasmus nach der Kontrastmittelapplikation, sodass eine Pharmakoangio- 


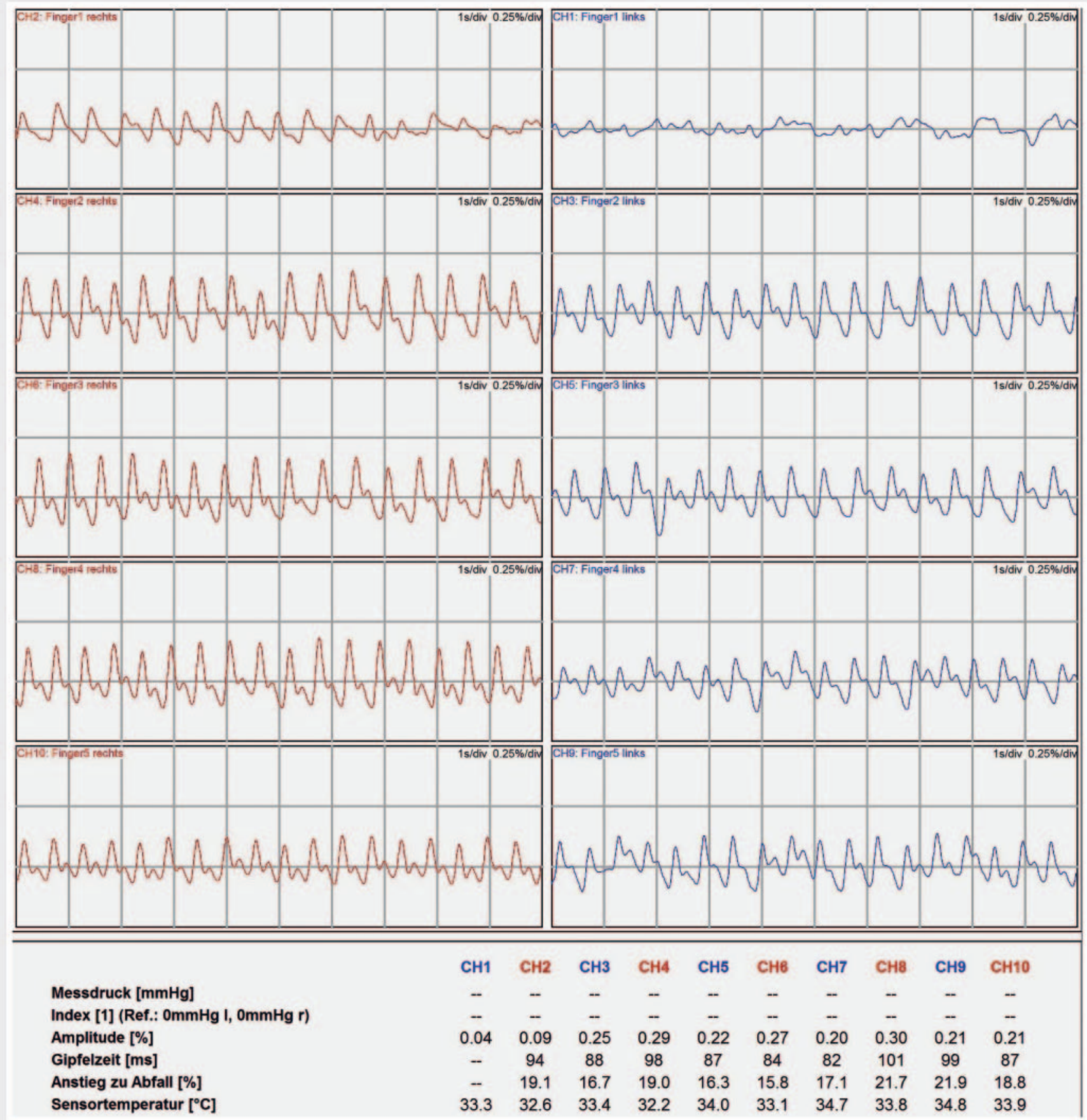

- Abb.3 Akrale optische Pulswellensoszillografie (OPO, AngE-System Fa SYCO MeData GmbH) der Finger (rot = rechte Hand, blau = linke Hand), Patient mit nachgewiesenem Hypothenar-Hammer-Syndrom rechts. OPO mit seitengleichem, unauffälligem Befund (D1 links reduzierte Pulsatilität, DD Störartefakt).

grafie nötig ist. Die vermutete Grunderkrankung muss weitere diagnostische Schritte nach sich ziehen ( $\triangleright$ Tab. 2). Organische Durchblutungsstörungen ohne kritische Extremitätenischämie machen eine sofortige Intervention/Operation nicht notwendig.

\section{Funktionelle Durchblutungsstörung}

Liegt keine organische Durchblutungsstörung vor, bleibt Zeit für eine detaillierte differenzialdiagnostische Abklärung. Während bei kritischer organischer Durchblutungsstörung die Anamneseerhebung zunächst symptomorientiert erfolgen kann, ist bei funktionellen Durchblutungsstörungen (organisch kompensierten Durchblutungsstörungen) die Anamnese maßgeblich für die weiteren diagnostischen Schritte. Es gibt 4 wesentliche Entitäten, die in der differenzialdiagnostischen Abklärung eine Rolle spielen: das Thoracic-outlet-Syndrom (TOS), das Karpaltunnelsyndrom, 
- Tab. 2 Weiterführende diagnostische Verfahren bei organischen Durchblutungsstörungen der Hand.

\begin{tabular}{|l|l|}
\hline ursächliche Erkrankung & diagnostische Auswahl \\
\hline PAVK & $\begin{array}{l}\text { Anamnese, Screening nach KHK } \\
\text { und CAVK }\end{array}$ \\
\hline Thrombangiitis obliterans & $\begin{array}{l}\text { Anamnese, Screening Beindurchblu- } \\
\text { tung }\end{array}$ \\
\hline arterielle Embolie & $\begin{array}{l}\text { LZ-EKG, ggf. transösophageale } \\
\text { Echokardiografie }\end{array}$ \\
\hline
\end{tabular}

das primäre Raynaud-Phänomen und andere Ursachen für ein sekundäres Raynaud-Phänomen.

Unter Vibrationsbelastung treten Nervenschädigungen als Kribbelparästhesien oder Taubheitsgefühl zunächst nach Vibrationsbelastung und später auch spontan auf. Sie werden in der Regel 3 Jahre vor den vaskulären Beschwerden bemerkt und betreffen meist alle Finger [26]. Ein spezieller Befall mit Zugehörigkeit zu einem Nervenversorgungsgebiet ist verdächtig auf ein Karpaltunnelsyndrom oder kann auch Hinweise auf eine Läsion des N. ulnaris in der Guyon-Loge (DD-Kompression durch ein Aneurysma $\operatorname{der}$ A. ulnaris) geben. Die vaskulären Veränderungen treten als Raynaud-Phänomen in Erscheinung und können im Anfall auch mit Parästhesien, Taubheit und Schmerzen einhergehen. Eine Daumenbeteiligung spricht gegen ein primäres Raynaud-Phänomen. Klassischerweise zeigt das Raynaud-Phänomen eine Tricolore-Symptomatik. In der wissenschaftlichen Literatur werden biphasische Verläufe (am häufigsten Abblassung und Zyanose) oder sogar auch das alleinige Abblassen der Finger als Raynaud-typisch bewertet [27, 28]. Sind nur einzelne Finger betroffen oder fehlt eine Hyperämiephase, deutet dies stark auf eine organische Durchblutungsstörung hin. Da oft eine RaynaudSymptomatik angegeben wird, jedoch bei Vorstellung überwiegend nicht präsentiert werden kann, helfen Fotodokumentationen. Es sollte darauf geachtet werden, dass diese die Handrücken und die Handinnenflächen bei erhobenen Händen (zur Personenidentifikation) abbilden. Meist können diese erst bei der zweiten Konsultation dementsprechend gesichtet werden.

Klinische Tests zum Ausschluss eines Karpaltunnelsyndroms (Phalen-, Tinel-Test) müssen immer erfolgen. Eine Messung der Nervenleitgeschwindigkeit von Nervus medianus und Nervus ulnaris ist in der Regel bei funktionellen Durchblutungsstörungen ratsam.

Das Thoracic-outlet-Syndrom sollte neben klinischen Testungen (Roos-, Adson- und Halstead-Test) auch mit Funktionsduplexsonografien oder akralen optischen Funktionspulsoszillografien objektiviert werden. Hilfreich sind hierbei oft Unterstützungen in der Testdurchführung durch Kollegen der physikalischen Medizin oder Physiotherapie.

Die Abklärung eines sekundären Raynaud-Phänomens beinhaltet meist eine weitreichende laborchemische Diagnostik (Kollagenosen, rheumatoide Arthritis etc.) und Tumorsuche (z. B. Plasmozytome, solide Tumoren etc.). Aber auch Nebenwirkungsprofile von Medikamenten (Ergotamine etc.) oder andere Berufsexposi- tionen müssen bedacht werden (Vinylchlorid-Krankheit). Der positive prädiktive Wert für das Vorliegen einer Kollagenose ist allein durch die Kapillarmikroskopie höher als durch die Laborwertbestimmung. Die generell mangelnde Verfügbarkeit der Methode kann durch die fehlende Abrechnungsmöglichkeit bei hohem zeitlichem Aufwand begründet sein.

\section{Spezielle Abklärung bei vibrationsinduzierten Schäden}

Für vibrationsbedingte Gefäß- und Nervenschädigungen gibt es aus der Arbeitsmedizin spezielle Untersuchungsmethoden, die einem normierten Ablauf unterliegen und standardisiert ausgewertet werden können. Gibt es Hinweise auf einen vibrationsbedingten Gefäß- oder Nervenschaden, sollten die folgenden Untersuchungen erfolgen:

1. Abblassungs-Score nach Griffin ( $\triangleright$ Abb. 4, vaskuläre Komponente)

2. Kältebelastungstest (standardisiert nach DIN ISO 148351:2016 Abkühlung auf $12^{\circ} \mathrm{C}$ über 5 Minuten) für vaskuläre Komponente

a) systolische Fingerdruckmessung vor und nach Kältebelastung (nach DIN ISO 14835-2:2005)

b) Thermometrie mit Wiedererwärmungszeit nach Kältebelastung (nach DIN ISO 14835-1:2016)

c) Thermografie nach Kältebelastung (nicht standardisiert)

3. Sensibilitätsprüfung für nervale Komponente

a) Druckempfinden mit dem Semmes-Weinstein-Monofilament oder mit Stimmgabel (Rydell-Seiffert)

b) Pallästhesiometrie mit $31,5 \mathrm{~Hz}$ oder $125 \mathrm{~Hz}$ (nach DIN ISO 13091-1/2:2003)

c) Quantitativ sensorische Testung

Die Ergebnisse der vaskulären und nervalen Testung führen zu einer Schweregradbeurteilung. Im Jahr 2018 wurden durch ein internationales Konsortium Kriterien definiert, die den Schweregrad des Vibrationssyndroms der Finger beschreiben ( $\downarrow$ Tab. 3). Die vaskuläre Komponente wurde nur anhand des klinischen Kriteriums der Abblassung graduiert. Die standardisierten Funktionstestungen für die vaskuläre Komponente dienen jedoch exzellent dazu, die funktionelle Vasokonstriktion zu provozieren.

\section{Therapeutische Optionen}

\section{Vorgehen beim Hypothenar-/Thenar-Hammer-Syndrom}

Konservative Therapiemaßnahmen bei HHS/THS-Patienten wurden überwiegend in den vorliegenden Fall-Kontroll-Untersuchungen erfolgreich angewandt $[30,31]$. In den publizierten Fallberichten scheiterten konservative Therapiestrategien hingegen oft. Bei fehlenden Hinweisen auf eine kritische Ischämie scheint auch bei darstellbaren segmentalen Okklusionen eine konservative Therapie empfehlenswert [32]. Neben der therapeutischen Antikoagulation mit Vitamin-K-Antagonisten, Heparin oder NOAK bzw. Thrombozytenaggregationshemmung (ASS, Clopidogrel, Calciumcarbasalt) werden verschiedene vasoaktive Substanzen wie Prostaglandin E1 Aloprostadil, das Prostacyclin-Analogon 


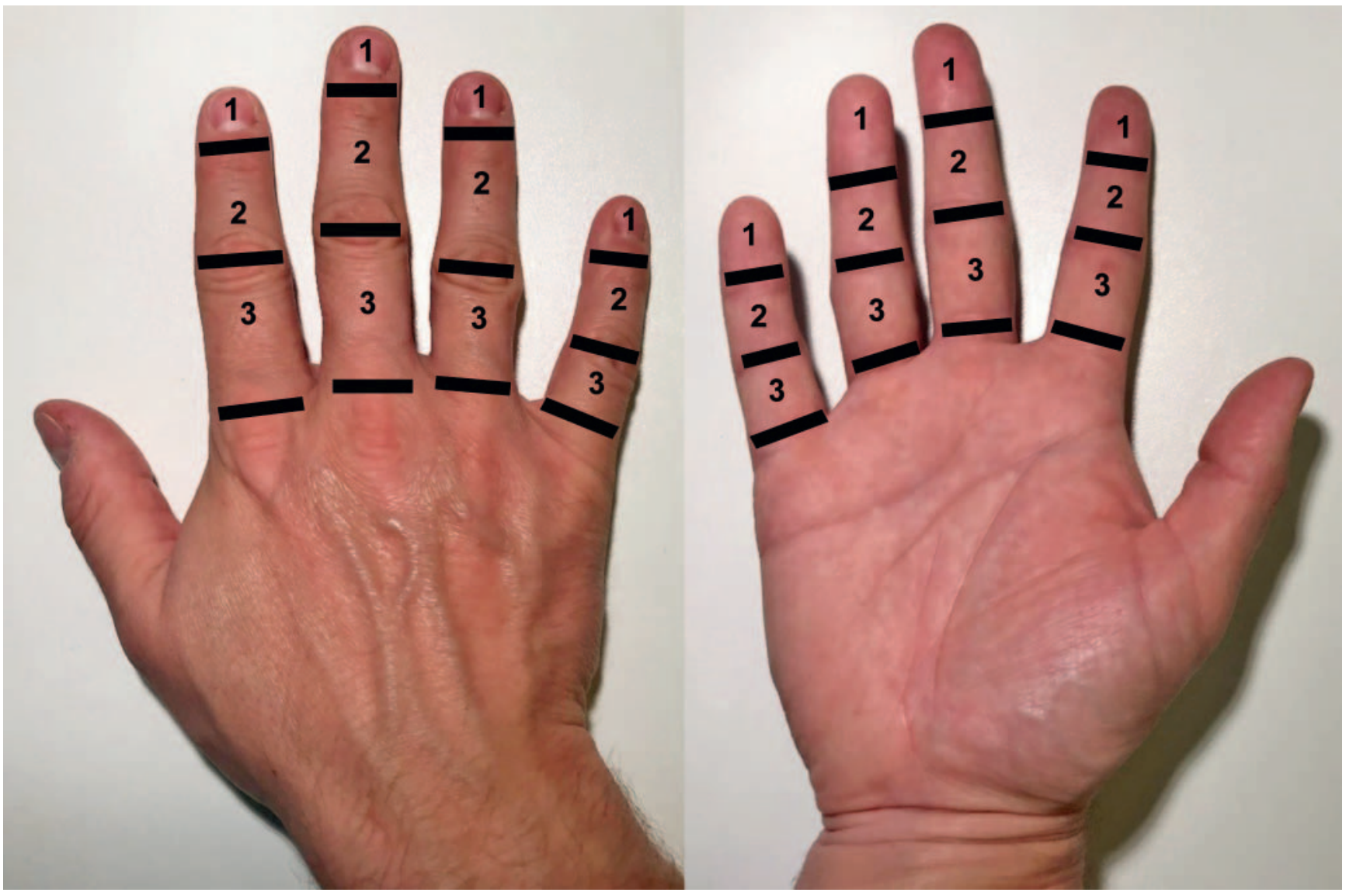

- Abb.4 Darstellung der rechten Hand mit möglichen Score-Werten (Griffin-„Ablassungs-Score“): Abblassung und Hyperämie oder Abblassung und Zyanose sind klinische Symptome, die eine Punkteerfassung möglich machen (Maximalwert 24 Punkte pro Hand).

Iloprost und Fibrinolytika wie Alteplase in der wissenschaftlichen Literatur erwähnt. Außerdem wurde mit Pentoxifyllin, KalziumAntagonisten vom Dihydropyridin- und Benzothiazepin-Typ sowie dem Alpha-Adrenorezeptor-Antagonisten Buflomedil behandelt [33-37].

Endovaskuläre Verfahren, v. a. die lokale Lysetherapie, sind Bestandteil eines multimodalen Therapiekonzepts und folgten in den Fallbeschreibungen zumeist gescheiterten konservativen Therapiemaßnahmen bei Patienten mit HHS. Lokale Lysetherapien wurden mit Erfolgsraten von bis zu $46 \%$ beschrieben [38]. Untersuchungen und Berichte zur Anwendung von Drug-ElutingBallons sind noch Raritäten. Eine Stentimplantation scheint im Bewegungssegment prognostisch nicht sinnvoll zu sein. FogartyManöver wurden vereinzelt beschrieben.

Gefäßchirurgische Eingriffe mit Resektion pathologischer Segmente und folgender End-zu-End-Anastomose oder Venen-Arterien-Interposition dominierten in der Literatur. Alleinige Ligaturen des pathologischen Gefäßsegments sowie lokale Rekonstruktionen kommen selten zum Einsatz. Yuen et al. erarbeiteten einen pathologiespezifischen Workflow: nach gescheiterter konservativer Therapie und erfolgloser Katheter-gestützter Fibrinolyse sollte das thrombosierte Segment reseziert werden. In Abhängigkeit vom Abstrom in den Fingerbereich und von der Länge des resezierten pathologischen Gefäßsegments erfolgte eine direkte Anastomose oder eine Gefäßinterposition. Eine Ligatur ohne Herstellung der Ge- fäßkontinuität war nur bei Fehlen von Ischämiezeichen zulässig [32]. Betrug das resezierte Gefäßsegment weniger als $2 \mathrm{~cm}$ und war der oberflächige Hohlhandbogen kompetent, erfolgte eine direkte Gefäßanastomose. Bei aneurysmatischen Gefäßveränderungen sollte ein chirurgisches Vorgehen in Betracht gezogen werden. Sind diese thrombosiert, kann bei kritischer Ischämie zunächst einer lokalen Lysetherapie erfolgen [32]. Material der Gefäßinterposition waren vorwiegend körpereigene Venen (V. saphena magna, V. cephalica, V. basilica, Fußrückenvene). Die Offenheitsraten variierten in Abhängigkeit der Arbeitsgruppe.

Für die thorakale Sympathektomie gibt es keine konsistente Datenlage. Eine Arbeitsgruppe untersuchte Daten von insgesamt 728 Anwendungen (endoskopische transthorakale Sympathektomie, perkutane Radiofrequenz-Sympathektomie und offene thorakale Sympathektomie) bei Patienten mit Fingerischämien (primäres und sekundäres Raynaud-Phänomen). Ein positiver Effekt wurde postoperativ bei $92 \%$ der Patienten mit primärem und bei $89 \%$ der Betroffenen mit sekundärem Raynaud-Phänomen gemessen. Der Langzeiteffekt (bis zu 17 Jahre) war vor allem beim primären Raynaud-Phänomen mit 58\% deutlich schlechter als in der Gruppe mit sekundärem Raynaud-Phänomen (89\%). Eine Ulkusabheilung war in $95 \%$ der Fälle festzustellen [39]. Im Langzeit-Follow-up bei HHS-Patienten kamen Dethmers et al. zu dem Ergebnis, dass die thorakale Sympathektomie keine Therapieverbesserung zur alleinigen Gefäßinterposition erbrachte [40]. 
- Tab. 3 Stadieneinteilung der vaskulären und neurologischen Komponente nach internationalen Konsensus-Kriterien (ICC 2018) [modifiziert nach [29]].

\section{vaskuläre Komponente}

(nach Griffin-Score, max. 24 Punkte)

\begin{tabular}{l|l} 
ICC-Stadium $\quad$ Symptome \\
\hline
\end{tabular}

\begin{tabular}{|l|l|}
\hline $0 \mathrm{~V}$ & keine Abblassungsattacken \\
\hline $1 \mathrm{~V}$ & Griffin-Abblassungs-Score 1-4 \\
\hline
\end{tabular}

2 V Griffin-Abblassungs-Score 5-12

$3 \mathrm{~V}$

\section{neurologische Komponente}

(anhaltende Symptomatik > 20 Minuten, 2 von 3 standardisierte Verfahren (Semmes-Weinstein-Monofilament, Vibrationswahrnehmung, Temperaturwahrnehmung), mind. ein Finger im Medianus- und Ulnarisversorgungsgebiet)

\section{ICC-Stadium Symptome}

$0 \mathrm{~N}$

$1 \mathrm{~N}$

$2 \mathrm{~N}$

$3 N$

\begin{abstract}
kein Taubheitsgefühl oder Kribbeln der Finger
intermittierendes Taubheitsgefühl und/oder Kribbeln der Finger

intermittierendes Taubheitsgefühl und/oder Kribbeln der Finger + Verlust der sensorischen Wahrnehmung in mindestens 1 Finger; nachgewiesen durch 2 oder mehr valide Methoden: Druckwahrnehmung, Temperaturwahrnehmung, Vibrationswahrnehmung

intermittierendes Taubheitsgefühl und/oder Kribbeln der Finger + Verlust der sensorischen Wahrnehmung in mindestens 1 Finger; nachgewiesen durch 2 oder mehr valide Methoden: Druckwahrnehmung, Temperaturwahrnehmung, Vibrationswahrnehmung + Symptome und objektiver Nachweis der beeinträchtigten Fingerfertigkeit (Purdue-pegboard-Test)
\end{abstract}

\section{Vorgehen bei vibrationsbedingten Durchblutungsstörungen}

Ein sekundäres Raynaud-Phänomen kann über die Behandlung der grundlegenden Erkrankung minimiert werden. Dies bedeutet bei vibrationsinduzierten Durchblutungsstörungen die Expositionsreduktion. Das Meiden von Vibrationswerkzeugen, nasskaltem Wetter oder windigen Wetterlagen kann vasospastische Anfälle minimieren. Hierzu ist eine Optimierung des Arbeitsplatzes, der Arbeitsabläufe und ggf. auch der Arbeitsgeräte nach Analyse durch den Arbeitgeber nötig. Sauni et al. zeigten in einer Umfrage unter Patienten mit Vibrationskrankheit, dass 8,5 Jahre nach Diagnosestellung etwa jeder dritte Befragte eine Besserung der vaskulären und sensoneurologischen Beschwerden bemerkte. Je kürzer die berufliche Vibrationsexposition war, desto höher war die Wahrscheinlichkeit der Symptomminderung. Eine Veränderung des Arbeitsplatzes wurde nur sehr selten praktiziert [41]. Dupuis et al. sahen 2-6 Jahre nach Expositionsstopp bei 54\% eine Verbesserung der Beschwerden, nur bei $7 \%$ zeigte sich der Befund aggraviert [42]. Der Gebrauch von vibrations- und schlagdämpfenden Handschuhen kann den Vibrationseffekt um fast $86 \%$ reduzieren [43].

Komplementäre und alternative Therapieformen wie Akkupunktur und Biofeedback sowie die Einnahme von essenziellen Fettsäuren, Ginkgo-Extrakten, Antioxidantien, L-Arginin und Glukosaminoglykanen, der Gebrauch von speziellen Handschuhen sowie die Low-Level-Lasertherapie bei Raynaud-Patienten wurden in einer Metaanalyse von 20 randomisierten klinischen Studien von Malenfant et al. untersucht. Aufgrund des schlechten Studiendesigns konnte keine abschließende Beurteilung erfolgen [44]. Ein apparatives Gefäßtraining (z. B. Knetbälle oder Finger-Expander) kann die periphere Hämodynamik verbessern.

Konservative Therapieversuche bei vibrationsbedingten Durchblutungsstörungen mit vasodilatierenden Medikamenten werden regelmäßig angewandt. Eine Evidenz liegt nicht vor. Medikamentöse Behandlungsversuche können mit Kalziumantagonisten vom Dihydropyridin- und Diltiazem-Typ durchgeführt werden. Ebenso begrenzt ist die Datenlage für den Einsatz des Alpha-1Adrenorezeptorantagonisten Prazosin, des Angiotensin-Rezeptorblockers Lorasartan und des selektiven Serotonin-Wiederaufnahmehemmers Fluoxetin [45]. Salben mit hoher Nitratkonzentration ( $\geq 2 \%$, internationale Apotheke) sind häufig angewandte Topika im Raynaud-Anfall. Der Phosphodiesterase-5-Inhibitor Sildenafil und der Endothelin-Rezeptorantagonist Bosentan werden bei Sklerodermie eingesetzt und fanden bisher bei HHS/THS oder Vibrationssyndromen nicht eingesetzt [46]. Die Gabe von Aspirin 75-100 mg wurde nicht ausreichend untersucht und ist nur bei gleichzeitig bestehender PAVK einsetzbar [45]. Eine Modifikation kardiovaskulärer Risikofaktoren, insbesondere der Nikotinkarenz, kann sowohl bei vibrationsinduzierten Durchblutungsstörungen als auch beim HHS/THS das Krankheitsbild positiv beeinflussen.

Endovaskuläre und gefäßchirurgische Eingriffe kommen bei dieser Patientengruppe nicht infrage. Eine thorakale Sympathektomie sollte nur bei schwere Anfallssymptomatik durchgeführt werden.

\section{Funktionseinschränkung und Begutachtung}

Um eine Funktionseinschränkung der Hand bei funktionellen oder organischen Durchblutungsstörungen vorzunehmen, ist eine Beurteilung der anamnestischen und objektivierbaren apparativen Befunde nötig. Besteht eine große Diskrepanz muss an eine Aggravation gedacht werden.

Entsprechende Möglichkeiten zur Beurteilung der Fingerfertigkeit im Alltag mit dazugehörigem Berufs-/Freizeitmodul bietet der DASH-Fragebogen (DASH = Disabilities of the Arm, Shoulder and Hand). Dieser kann auch als Verlaufsdiagnostikum genutzt werden. Neuropsychologische Assessments, wie der Purdue-pegboard-Test 
oder der Nine-hole-peg-Test etc., objektivieren die Fingerfertigkeit. Es ist darauf hinzuweisen, dass die Handkraftmessung mit einem Handdynamometer nur die grobe Krafteinschränkung der Hand misst und lange Zeit nach Erkrankungsbeginn ohne Pathologien bleibt. Bei auffälligen Testergebnissen sollte stets nach anderen Ursachen wie kognitiver Beeinträchtigung oder anderen neurologischen Erkrankungen gefahndet werden.

Die messbare Funktionseinschränkung ist Grundlage für die Bewertung der Minderung der Erwerbsfähigkeit (MdE). Explizite MdE-Empfehlungen liegen nicht vor. In Deutschland sind vibrationsbedingte Durchblutungsstörungen seit 1979 unter der Nummer BK 2104 anerkannt, wogegen das Hypothenar-HammerSyndrom (BK 2114) erst seit dem Jahr 2015 als entschädigungsrelevante Berufskrankheit gilt [47, 48]. Jeder Arzt hat laut § 202 SGB VII die Pflicht, den Verdacht auf eine Berufskrankheit der Unfallversicherung mit den entsprechenden Formularen anzuzeigen.

Berufsgruppen mit besonderer Exposition für HHS/THS und vibrationsbedingte Schädigungen im Finger- und Handbereich sind Dachdecker, Zimmermänner, Kfz-Mechaniker, Möbeltransporteure, Installateure, Schreiner, Mechaniker, Elektriker, Maschinisten, Forstarbeiter, Gärtner, Bergleute oder Steinbohrer sowie auch Landwirte, da sie häufig handgehaltene Vibrationsgeräte einsetzen und ihre Hand als Hammerersatz einsetzen [49, 50]. Bestimmte Sportarten wie Handball, Schlägersportarten, Kampfsportarten, Frisbeewerfen oder auch Sportarten, bei denen der Handballen in fixierter Position verweilt (Fahrrad-/Motorradfahren), erhöhen das Risiko für ein HHS [51]. Deren Identifikation ist bei versicherungsrechtlichen Fragestellungen von großer Bedeutung.

Voraussetzung für die Anerkennung des HHS/THS als BK 2114 ist die Trias aus Nachweis einer schädigenden Einwirkung, den typischen Gefäßveränderungen und dem Ausschluss anderer Ursachen. Häufig liegen schnittbildgebende Verfahren oder angiografische Bilder durch vorangegangene Therapien vor. Die Schwierigkeit in der Begutachtung liegt darin, den kausalen Zusammenhang zwischen Exposition und Erkrankung zu begründen. Die einmalige stumpfe Gewalteinwirkung mit nachweisbarer Gefäßläsion reicht zur Anerkennung aus. Eine Dosis-WirkungsBeziehung besteht nicht $[48,49]$. Nach Fingeramputation dienen die gutachterlichen Standardwerke als Hilfe zur Bemessung der individuellen MdE.

Bei vibrationsbedingten Krankheitserscheinungen bei BK 2104 liegt die Schwierigkeit im Nachweis der Erkrankung (Vollbeweis). Über den Technischen Aufsichtsdient der Berufsgenossenschaften liegen meist ausführliche Befunde zur beruflichen Exposition vor. Es wird angenommen, dass nur eine dauerhafte Vibrationsexposition über Monate bis Jahre eine Gefäß- und Nervenschädigung auslöst.

Ein Unterlassungszwang ist für die Anerkennung der BK 2104 nötig. Somit kann die Berufserkrankung nur anerkannt werden, wenn die berufliche Tätigkeit unterlassen wird. Je kürzer die berufliche Vibrationsexposition ist, umso wahrscheinlicher ist auch eine Symptomminderung durch Vibrationskarenz. In der wissenschaftlichen Literatur gibt es Untersuchungen der universitären Arbeitsmedizin in Mainz, die zu wertvollen Anhaltspunkten für die gutachterliche Bewertung geführt haben (Staffelung 10, 20 und 30 von 100) [52].

\section{Zusammenfassung}

Patienten mit Beschwerden an den oberen Extremitäten und insbesondere im Handbereich sind keine Seltenheit in der gefäßmedizinischen Praxis. Neben Venen- und Lympherkrankungen spielen arterielle Durchblutungsstörungen eine Rolle. Oft müssen sie unter differenzialdiagnostischer Absicht bei chirurgischen Krankheitsbildern im muskuloskelettalen Bereich oder dem Karpaltunnelsyndrom ausgeschlossen werden. Es dominieren Kribbelparästhesien und das Raynaud-Phänomen. Neben den bekannten Ursachen müssen das Hypothenar-/Thenar-Hammer-Syndrom und das Vibrationssyndrom der Finger ausgeschlossen werden, da deren Diagnose auch versicherungsrechtliche Konsequenzen hat. Das diagnostische Hauptaugenmerk beim Hypothenar-/Thenar-HammerSyndrom liegt im bildmorphologischen Nachweis der Gefäßläsion, wohingegen beim Vibrationssyndrom der Finger vaskuläre und nervale Pathologien mit Provokationstests objektiviert werden müssen. Den maßgeblichen Therapieansatz beim Vibrationssyndrom der Finger stellt die Expositionsprophylaxe dar. Medikamentöse Maßnahmen können sich an denen des sekundären Raynaud-Phänomens orientieren. Das Hypothenar-/Thenar-Hammer-Syndrom kann mit einer kritischen Extremitätenischämie einhergehen. In diesen Fällen ist eine Lumen-eröffnende Maßnahme erforderlich. Überwiegend werden in der Literatur hierfür Bypassoperationen angeführt. In der Begutachtung muss die anamnestisch angegebene Funktionseinschränkung mit den objektivierbaren Parametern abgeglichen werden. Die Bemessung der MdE ist stets individuell zu treffen. Hilfreich kann ein interdisziplinärer Austausch mit den Kollegen der Handchirurgie oder Ergotherapie sein. In der gefäßmedizinischen Praxis sollte eine Grundkenntnis dieser Entitäten bestehen. Wenn ein Zusammenhang zwischen den Gefäßpathologien und der beruflichen Tätigkeit wahrscheinlich ist, gilt nach § 202 Sozialgesetzbuch VII die Verpflichtung für jeden Arzt, den Berufskrankheitsverdacht zur Anzeige zu bringen.

Interessenkonflikt

Die Autorinnen/Autoren geben an, dass kein Interessenkonflikt besteht.

Literatur

[1] Wahl U, Kaden I, Köhler A et al. Vascular trauma of the hand - a systematic review. VASA 2019; 48: 205-215

[2] Heidrich H. Functional vascular diseases: Raynaud's syndrome, acrocyanosis and erythromelalgia. Vasa 2010; 39: 33-41

[3] Marie I, Hervé F, Primard E et al. Long-term follow-up of hypothenar hammer syndrome: a series of 47 patients. Medicine (Baltimore) 2007; 86: 334-343

[4] Vayssairat M, Debure C, Cormier JM et al. Hypothenar hammer syndrome: Seventeen cases with long-term follow-up. J Vasc Surg 1987; 5: 838-843

[5] Taute BM, Behrmann C, Cappeller WA et al. Das sonografische Bild des Hypothenar-Hammer-Syndroms. Ultraschall in Med 1998; 19: 220-224

[6] Griffin M]. Frequency-dependence of psychophysical and physiological response to hand-transmitted vibration. Ind Health 2012; 50: 354-369

[7] Dong RG, Welcome DE, McDowell TW et al. A Proposed Theory on Biodynamic Frequency Weighting for Hand-Transmitted Vibration Exposure. Ind Health 2012; 50: 412-424 
[8] Bundesministerium für Gesundheit (BMGS). Merkblatt zur Berufskrankheit Nr. 2103 der Anlage der Berufskrankheiten-Verordnung (BKV). BArbBI 2005; 3: 51

[9] Herrick AL. Pathogenesis of Raynaud's phenomenon. Rheumatology 2005; 44: 587-596

[10] Stoyneva Z, Lyapina M, Tzvetkov D et al. Current pathophysiological views on vibration-induced Raynaud's phenomenon. Cardiovasc Res 2003; 57: 615-624

[11] Matoba T. Pathophysiology and clinical picture of hand-arm vibration syndrome in Japanese workers. Nagoya J Med Sci 1994; 57: 19-26

[12] Kanazuka M, Shigekiyo T, Toibana N et al. Increase in plasma thrombomodulin level in patients with vibration syndrome. Thromb Res 1996; 82: $51-56$

[13] Palmer KT, Mason H. Serum endothelin concentrations in workers exposed to vibration. Occup Environ Med 1996; 53: 118-124

[14] Popević MB, Janković SM, Borjanović SS et al. Assessment of coarse and fine hand motor performance in asymptomatic subjects exposed to hand-arm vibration. Arh Hig Rada Toksikol 2014; 65: 29-36

[15] Heaver C, Goonetilleke KS, Ferguson $\mathrm{H}$ et al. Handarm vibration syndrome: a common occupational hazard in industrialized countries. J Hand Surg Eur 2011; 36: 354-363

[16] European Agency for Safety and Health at work. Workplace exposure to Vibration in Europe: an expert review 2008. Im Internet (Stand: 18.02.2020): http://www.osha.europa.eu

[17] Su AT, Darus A, Bulgiba A et al. The clinical features of hand-arm vibration syndrome in a warm environment-a review of the literature. J Occup Health 2012; 54: 349-360

[18] Bovenzi M. A prospective cohort study of exposure-response relationship for vibration-induced white finger. Occup Environ Med 2010; 67: $38-46$

[19] Letz R, Cherniack MG, Gerr F et al. A cross sectional epidemiological survey of shipyard workers exposed to hand-arm vibration. $\mathrm{Br}$ ] Ind Med 1992; 49: 53-62

[20] McGeoch KL, Gilmour WH. Cross sectional study of a workforce exposed to hand-arm vibration: with objective tests and the Stockholm workshop scales. Occup Environ Med 2000; 57: 35-42

[21] Diehm C, Allenberg JR, Nimura-Eckert K. Kapitel 8: Das Buerger-Syndrom. In: Diehm C, Hrsg Farbatlas der Gefäßkrankheiten. Nachdruck. Heidelberg, Berlin: Springer; 2004: 207-219

[22] Ketha SS, Cooper LT. The role of autoimmunity in thromboangiitis obliteras (Buerger's disease). Ann N Y Acad Sci 2013; 1258: 15-25

[23] Klein-Weigel PF, Volz TS, Zange L et al. Buerger's disease: providing integrated care. J Multidiscip Healthc 2016; 9: 511-518

[24] Klein-Weigel PF, Richter JG. Thromboangiitis obliterans (Buerger's diesease). Vasa 2014; 43: 337-346

[25] Ruland O, Borkenhagen N, Prien T. Der Doppler-Hohlhand-Test. Ultraschall in Med 1988; 9: 63-66

[26] Nilsson T, Wahlström J, Burström L. Handarm-vibration and the risk of vascular and neurological diseases - A systematic review and metaanalysis. PLoSONE 2017; 12: e180795

[27] Belch J, Carlizza A, Carpentier PH et al. ESVM guidelines - the diagnosis and management of Raynaud's phenomenon. Vasa 2017; 46: 413-423

[28] Maverakis E, Patel F, Kronenberg DG et al. International consensus criteria for the diagnosis of Raynaud's phenomenon. J Autoimmun 2014; 48-49: 60-65

[29] Poole CJM, Bovenzi M, Nilsson T et al. International consensus criteria for diagnosing staging hand-arm vibration syndrome. Int Arch Occup Environ Health 2019; 92: 117-127

[30] Röhrl T. Das Hypothenar-Hammer-Syndrom. Ergebnisse einer multizentrischen Fall-Kontroll-Studie zur Erfassung beruflicher und außerberuflicher Risiken. 2008. Dissertation, LMU München
[31] Marie I, Hervé F, Primard E et al. Long-term follow-up of hypothenar hammer syndrome: a series of 47 patients. Medicine (Baltimore) 2007; 86: 334-343

[32] Yuen JC, Wrigth E, Johnson LA et al. Hypothenar Hammer Syndrome. Ann Plast Surg 2011; 76: 429-438

[33] Ablett CT, Hackett LA. Hypothenar hammer syndrome: case reports and brief review. Clin Med Res 2008; 6: 3-8

[34] Youakim S. Thenar hammer syndrome: a case report. Occup Med (Lond) 2006; 56: 507-509

[35] Smith HE, Dirks M, Patterson RB. Hypothenar hammer syndrome: Dista ulnar artery reconstruction with autologous inferior epigastric artery. J Vasc Surg 2004; 40: 1238-1242

[36] Sharma R, Ladd W, Chaisson G et al. Images in cardiovascular medicine: Hypothenar hammer syndrome. Circulation 2002; 105: 1615-1616

[37] Van de Walle PM, Moll FL, De Smet AA. The hypothenar hammer syndrome: update and literature review. Acta Chir Belg 1998; 98: 116-119

[38] Cejna M, Salomonowitz E, Wohlschlager $\mathrm{H}$ et al. Rt-PA thrombolysis in acute thromboembolic upper- extremity arterial occlusion. Cardiovasc Intervent Radiol 2001; 24: 218-223

[39] Coveliers HME, Hoexum F, Nederhoed JH et al. Thoracic sympathectomy for digital ischemia: a summary of evidence. J Vasc surg 2011; 54: 273-277

[40] Dethmers RSM, Houpt P. Surgical management of hypothenar and thenar hammer syndromes: a retrospective study a of 31 instances in 28 patients. J Hand Surg Br 2005; 30: 419-423

[41] Sauni R, Toivio P, Pääkkönen R et al. Work disability after diagnosis of hand-arm vibration syndrome. Int Arch Occup Environ Health 2015; 88: 1061-1068

[42] Dupuis H, Riedel S. Experience on the reversibility of the vibrationinduced white fi nger disease. Cent Eur J Public Health 1995; 3: 19-21

[43] Milosevic M, McConville KM. Evaluation of protective gloves and working techniques for reducing hand-arm vibration exposure in the workplace. J Occup Health 2012; 54: 250-253

[44] Malenfant D, Catton M, Pope JE. The efficacy of complementary and alternative medicine in the treatment of Raynaud's phenomenon: a literature review and meta-analysis. Rheumatology (Oxford) 2009; 48: 791-795

[45] Linnemann B, Erbe M. Raynaud's phenomenon and digital ischaemia pharmacologic approach and alternative treatment options. Vasa 2016 45: $201-212$

[46] Matucci-Cerinic M, Denton CP, Furst DE et al. Bosentan treatment of digital ulcers related to systemic sclerosis: results from the RAPIDS-2 randomised, double-blind, placebo-controlled trial. Ann Rheum Dis 2011; 70: 32-38

[47] Wahl U, Kaulbars U, Ernst F et al. Vibrationssyndrom der Finger - Eine Überarbeitung im Anerkennungsverfahren der BK 2104 ist nötig. Trauma Berufskrankh 2019; 21: 276-285

[48] Bundesministerium für Arbeit und Soziales (BMAS). Ärztliche Sachverständigenbeirat „Berufskrankheiten“ beim Bundesministerium für Arbeit und Soziales. (2012) Wissenschaftliche Begründung für die Berufskrankheit „Gefäßschädigung der Hand durch stoßartige Krafteinwirkung (HypothenarHammer-Syndrom und Thenar-Hammer-Syndrom)“. Bekanntmachung vom 1.5.2012 - IVa4-45226-2 GMBI. 6.6.2012: 449455. Im Internet (Stand: 19.02.2020): http://www.baua.de

[49] Scharnbacher J, Claus M, Reichert J et al. Hypothenar hammer syndrome: a multicenter case-control study. Am J Ind Med 2013; 56: 1352-1358

[50] De Monaco D, Frische E, Rigoni G et al. Hypothenar hammer syndrome. Retrospective study of nine cases. J Hand Surg Br 1999; 24: 731-734

[51] Scharnbacher ], Letzel S. Hypothenar-Hammer-Syndrom bei Sportlern. Deutsche Zeitschrift für Sportmedizin 2006; 57: 201-205

[52] Volter-Mahlknecht S, Gigic B, Muttray A et al. MdE-Verlauf und -Beurteilungbeim Vibrationsbedingten Vasospastischen Syndrom (VVS). Arbeitsmed Sozialmed Umweltmed 2010; 45: 582-591 